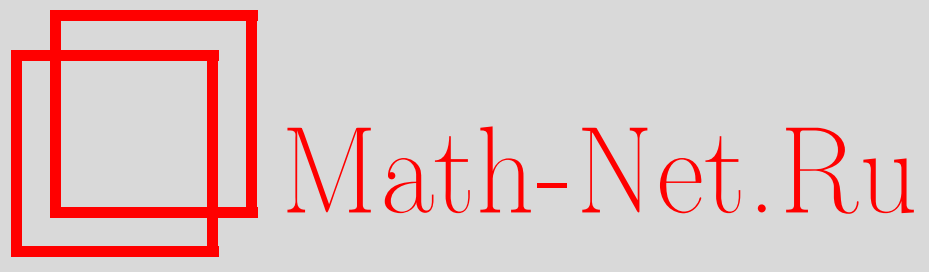

А. В. Королев, О сходимости неравномерных эргодических средних, Матем. заметки, 2010, том 87, выпуск $6,944-947$

DOI: https://doi.org/10.4213/mzm8748

Использование Общероссийского математического портала Math-Net.Ru подразумевает, что вы прочитали и согласны с пользовательским соглашением http://www . mathnet.ru/rus/agreement

Параметры загрузки:

IP : 54.197 .130 .99

26 апреля 2023 г., 14:36:21

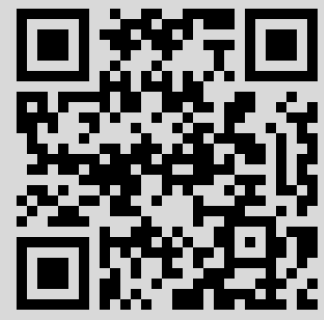




\section{О сходимости неравномерных эргодических средних}

\section{А. В. Королев}

Изучение неравномерных усреднений вида

$$
F_{t} f(x)=\int_{0}^{\infty} f\left(T_{t s} x\right) \nu(d s)
$$

для полупотоков $\left\{T_{t}\right\}$ с инвариантной мерой $\mu$ было начато в работах [1], [2], где было установлено, что для абсолютно непрерывных вероятностных мер $\nu$ на полуоси и ограниченных функций $f$ при $t \rightarrow+\infty$ величины $F_{t} f(x)$ для $\mu$-почти всех $x$ имеют тот же самый предел, что и в случае классического равномерного усреднения в теореме Бирхгофа-Хинчина. Исследование усреднений $F_{t} f(x)$ было продолжено в работе [3] (см. также [4; гл. 10]), где было выяснено, что для неограниченных функций $f$ может не быть сходимости неравномерных усреднений. Там же были получены дополнительные условия, дающие такую сходимость. Изучению аналогичных вопросов для стохастических уравнений посвящена работа [5]. Случай усреднений с операторной полугруппой рассмотрен в [6]. В данной работе исследуется вопрос о сходимости средних $F_{t} f(x)$ в $L^{p}(\mu)$, а также рассматриваются некоторые применения полученных результатов. В частности, речь идет об аналоге эргодической теоремы Винера-Винтнера. В отличие от результатов работ [3], [5], [6], в ряде утверждений этой работы наложено условие слабого перемешивания полугруппы.

Пусть $(X, \mathscr{A}, \mu)$ - вероятностное пространство. Напомним, что полугруппа $\left\{T_{t}\right\}_{t \geqslant 0}$ преобразований пространства $X$, сохраняющих меру $\mu$, называется слабо перемешивающей (см. [7; с. 28]), если для всяких двух функций $f, g \in L^{2}(\mu)$ выполнено равенство

$$
\lim _{t \rightarrow+\infty} \frac{1}{t} \int_{0}^{t}\left|\int_{X} f\left(T_{s} x\right) g(x) \mu(d x)-\int_{X} f d \mu \int_{X} g d \mu\right|^{2} d s=0 .
$$

В этом случае оператор $U_{t}$, действующий в $L^{1}(\mu)$ по формуле $U_{t} f(x)=f\left(T_{t} x\right)$, не имеет собственных функций, отличных от констант (см. [7; с. 35]).

Теорема 1. Пусть $\left\{T_{t}\right\}_{t \geqslant 0}$ - слабо перемешивающая полугруппа преобразований пространства $X$, причем соответствующая операторная полугруппа $U_{t}$ сильно непрерывна, а $\nu$ - произвольная вероятностная мера на $[0,+\infty)$, имеющая преобразование Фуръе $\widehat{\nu}$. Если $\widehat{\nu}(t) \rightarrow 0$ при $t \rightarrow+\infty$, то для всякой функции $f \in L^{p}(\mu)$ при $p \in[1,+\infty)$ выполнено равенство

$$
\lim _{t \rightarrow+\infty}\left\|\int_{0}^{+\infty} f\left(T_{t s} x\right) \nu(d s)-\int_{X} f(x) \mu(d x)\right\|_{L^{p}(\mu)}=0 .
$$

ДоказАтельство. Достаточно проверить доказываемое равенство для $f \in L_{p}(\mu)$ с нулевым интегралом. Сначала рассмотрим случай $p=2$. Применяя теорему Фубини и пользуясь теоремой Бохнера (см. [4; с. 146]), получим следующие равенства:

$$
\begin{aligned}
\left\|\int_{0}^{+\infty} f\left(T_{t s} x\right) \nu(d s)\right\|_{L^{2}(\mu)}^{2} & =\int_{X}\left[\int_{0}^{+\infty} \int_{0}^{+\infty} f\left(T_{t s} x\right) f\left(T_{t u} x\right) \nu(d s) \nu(d u)\right] \mu(d x) \\
& =\int_{0}^{+\infty} \int_{0}^{+\infty}\left[\int_{0}^{+\infty} e^{i t s \lambda} e^{-i t u \lambda} \sigma_{f}(d \lambda)\right] \nu(d s) \nu(d u) \\
& =\int_{0}^{+\infty}\left|\int_{0}^{+\infty} e^{i t s \lambda} \nu(d s)\right|^{2} d \sigma_{f}(d \lambda)
\end{aligned}
$$

Работа выполнена при поддержке Российского фонда фундаментальных исследований (гранты №№ 07-01-00536, 08-01-90431-Укр.). 
где $\sigma_{f}(\lambda)$ - спектральная мера функции $f$ относительно $T_{t}$. Мера $\sigma_{f}(\lambda)$ не имеет атомов, поскольку $T_{t}$ - слабо перемешивающая полугруппа, а среднее функции $f$ равно нулю (см. [7; с. 35]). Пользуясь условием $\widehat{\nu}(t) \rightarrow 0$ при $t \rightarrow+\infty$ и применяя теорему Лебега к последнему выражению предыдущего равенства, получаем справедливость равенства (1) для $p=2$. Случай $p=2^{k}, k>1$, проверяется аналогично. Для $1 \leqslant p<2^{k}$ доказываемое равенство следует из неравенства Гёльдера, ввиду которого норма в $L^{p}(\mu)$ мажорируется нормой в $L^{2^{k}}(\mu)$.

ЗАмечАниЕ 1. Из доказательства предыдущей теоремы видно, что условия на полугруппу $T_{t}$ можно ослабить, если вместо слабого перемешивания потребовать эргодичность полугруппы $T_{t}$ и отсутствие у спектральной меры $\sigma_{f}$ атома в нуле.

ЗАмечАние 2. Теорема 1 остается в силе в более общих ситуациях, например, когда полугруппа $T_{t}$ действует на пространстве $L^{1}(\mu)$ или в случае $n$-параметрической полугруппы.

Теперь рассмотрим некоторые случаи сходимости средних $F_{t} \mu$-почти всюду. Следующее предложение было рассмотрено для случая равномерных дискретных усреднений в $[8 ;$ с. 66$]$.

ПреДЛОЖениЕ 1. Пусть $\left\{f_{\alpha, \tau}, \alpha, \tau \in \mathbb{R}^{+}\right\}$- некоторое множество измеримых функиий на пространстве $X$ c $\sup _{\alpha, \tau}\left|f_{\alpha, \tau}\right| \in L^{p}(X)$, а $\varrho \in L^{q}(\lambda)$ - вероятностная плотность, имеющая ограниченный носитель в отрезке $[a, b]$. Пусть $\theta(t)=\underline{O}(t)$. Если при $\alpha, \tau \rightarrow+\infty$ ми имеем $f_{\alpha, \tau}(x) \rightarrow f(x)$ почти всюду, то

$$
\lim _{t \rightarrow+\infty} \int_{0}^{+\infty} f_{\Theta(t) s, t}\left(T_{t s} x\right) \varrho(s) d s=\mathrm{E}^{\mathscr{T}} f(x)
$$

для н-почти всех $x \in X$.

ДоказАтельство. В [3] было получено доказываемое равенство для $f_{\Theta(t) s, t}(x) \equiv$ $f(x)$, поэтому можно считать, что $f \equiv 0$. Проведем рассуждения для $\Theta(t)=t$, поскольку в общем случае рассуждения аналогичны. Имеем

$$
\begin{array}{r}
\limsup _{t \rightarrow+\infty}\left|\int_{0}^{+\infty} f_{t s, t}\left(T_{t s} x\right) \varrho(s) d s\right| \leqslant \limsup _{t \rightarrow+\infty}\left|\int_{0}^{r t^{-1}} f_{t s, t}\left(T_{t s} x\right) \varrho(s) d s\right| \\
+\limsup _{t \rightarrow+\infty}\left|\int_{r t^{-1}}^{+\infty} f_{t s, t}\left(T_{t s} x\right) \varrho(s) d s\right| .
\end{array}
$$

Положим $g_{r}=\sup _{\alpha \geqslant r, \tau \geqslant r}\left|f_{\alpha, \tau}\right|$. Тогда

$$
\begin{aligned}
\left|\int_{0}^{r t^{-1}} f_{t s, t}\left(T_{t s} x\right) \varrho(s) d s\right| & \leqslant\left(\int_{0}^{r t^{-1}} f_{t s, t}\left(T_{t s} x\right) d s\right)^{p^{-1}}\left(\int_{0}^{r t^{-1}} \varrho(s) d s\right)^{q^{-1}} \\
& \leqslant\left(\frac{1}{t} \int_{0}^{r} g_{0}\left(T_{s} x\right) d s\right)^{p^{-1}}\left(\int_{0}^{r t^{-1}} \varrho(s) d s\right)^{q^{-1}}
\end{aligned}
$$

при этом последнее выражение стремится к 0 при $t \rightarrow+\infty$. Пользуясь предыдущими оценками, получаем неравенство

$$
\begin{aligned}
\limsup _{t \rightarrow+\infty}\left|\int_{0}^{+\infty} f_{t s, t}\left(T_{t s} x\right) \varrho(s) d s\right| & \leqslant \limsup _{t \rightarrow+\infty}\left|\int_{r t^{-1}}^{+\infty} f_{t s, t}\left(T_{t s} x\right) \varrho(s) d s\right| \\
& \leqslant \lim _{t \rightarrow+\infty}\left|\int_{r t^{-1}}^{+\infty} g_{r}\left(T_{t s} x\right) \varrho(s) d s\right| \\
& \leqslant \lim _{t \rightarrow+\infty}\left|\int_{0}^{+\infty} g_{r}\left(T_{t s} x\right) \varrho(s) d s\right|=\mathrm{E}^{\mathscr{T}} g_{r}(x)
\end{aligned}
$$


где $\mathrm{E}^{\mathscr{T}} g_{r}$ есть условное математическое ожидание $f$ относительно $\sigma$-алгебры $T_{t}$-инвариантных множеств. Пользуясь тем, что $\int_{X} g_{r}(x) \mu(d x) \rightarrow 0$ при $t \rightarrow+\infty$, окончательно получаем, что

$$
\limsup _{t \rightarrow+\infty}\left|\int_{0}^{+\infty} f_{t s, t}\left(T_{t s} x\right) \varrho(s) d s\right|=0 .
$$

Предложение доказано.

В работе [9] (см. также [10]) был получен результат, обобщающий эргодическую теорему Биркгофа-Хинчина (см. [7; с. 17]), согласно которому для всякой эргодической полугруппы $T_{t}$ и интегрируемой функции $f \in L^{1}(\mu)$ найдется множество $A \in X$ с $\mu(A)=1$ такое, что при $x \in A$ и $\lambda \in[0,2 \pi)$ существует предел средних

$$
\frac{1}{T} \int_{0}^{T} e^{i \lambda s} f\left(T_{s} x\right) d s
$$

при $T \rightarrow+\infty$, причем если полугруппа $T_{t}$ является слабо перемешивающей и $\lambda \neq 0$, то этот предел равен 0. Рассмотрим средние

$$
F_{t, \lambda}=\int_{0}^{+\infty} e^{i \lambda t s} f\left(T_{t s} x\right) \nu(d s) .
$$

Приведем утверждение о сходимости средних $F_{t, \lambda}$, которое доказывается аналогично случаю усреднений Козлова-Трещева для эргодической теоремы Биркгофа-Хинчина (см. [3; теорема 3]).

ПредложениЕ 2. Пусть $T_{t}$ - слабо перемешивающая полугруппа. Тогда для всякой функции $f \in L^{p}(\mu)$ и всякой вероятностной плотности $\varrho \in L^{q}(\lambda)$ с ограниченным носителем на $[0,+\infty)$, где $p^{-1}+q^{-1}=1$ и $p, q \in[1,+\infty]$, найдется такое множество $A \in X$, что $\mu(A)=1$ и для всех $x \in A$ и $\lambda \in(0,2 \pi)$ выполнено равенство

$$
\lim _{t \rightarrow+\infty} \int_{0}^{+\infty} e^{i \lambda t s} f\left(T_{t s} x\right) \varrho(s) d s=0 .
$$

Рассмотрим теперь ситуацию, когда на вероятностном пространстве $(X, \mathscr{A}, \mu)$ определено измеримое отображение $T$, сохраняющее меру $\mu$. Пусть $g: X \rightarrow(0,+\infty)$ - измеримая функция. Напомним конструкцию, позволяющую построить полупоток $T_{t}$ на пространстве $\Omega_{g}$ под графиком $g$, сохраняющий меру $\mu_{g}$, полученную ограничением меры $\mu \otimes \lambda$ на $\Omega_{g}$, где $\lambda$ - мера Лебега. Полугруппа $T_{t}$ действует следующим образом: если $t \leqslant f(x)-s$, то $T_{t}(x, s)=(x, s+t)$, иначе отождествляют все точки вида $(y, f(y))$ и $(T y, 0)$ и полагают $T_{t}(x, s)=\left(T^{k} x, u_{k}\right)$, где

$$
u_{k}:=\sum_{i=1}^{k} f\left(T^{i}\right)-s-t, \quad k:=\min \left\{l: u_{l} \geqslant 0\right\} .
$$

Согласно результату Рудольфа (см. [8]) любой непериодический полупоток с точностью до метрического изоморфизма представляется как полупоток под графиком измеримой на $X$ функции, принимающей только два значения. Пусть $g \equiv 1$ на $X$ и $f$ - измеримая функция на $\Omega_{g}$. В случае, когда $f$ зависит только от $x \in X$, усреднения Козлова-Трещева выражаются в терминах дискретных неравномерных усреднений общего вида

$$
S_{n} f(x)=\sum_{k=0}^{+\infty} a_{k, n} f\left(T^{k} x\right) .
$$

Действительно, пусть $\varrho$ - вероятностная плотность на $[0,+\infty)$. Тогда выполнено равенство

$$
\int_{0}^{+\infty} T_{n s} f(x, \tau) \varrho(s) d s=\sum_{k=0}^{+\infty} T^{k} f(x) \int_{(k-\tau) / n}^{(k-\tau+1) / n} \varrho(s) d s .
$$


Проверим это равенство. В нашем случае $T_{t}(x, \tau)=\left(T^{[t+\tau]},\{t+\tau\}\right)$; значит, для всякого $k$ имеем

$$
T_{n s}(x, \tau)= \begin{cases}\left(T^{[n s]+1} x,(\tau+\{n s\}) \bmod 1\right), & \text { при } s \in\left(\frac{k-\tau}{n}, \frac{k}{n}\right), \\ \left(T^{[n s]} x,(\tau+\{n s\}) \bmod 1\right), & \text { при } s \in\left(\frac{k-1}{n}, \frac{k-\tau}{n}\right) .\end{cases}
$$

Тогда верны равенства

$$
\begin{aligned}
\int_{0}^{+\infty} T^{n s} f(x, \tau) \varrho(s) d s & =\sum_{k=1}^{+\infty}\left(\int_{(k-1) / n}^{(k-\tau) / n} T^{k-1} f(x) \varrho(s) d s+\int_{(k-\tau) / n}^{k / n} T^{k} f(x) \varrho(s) d s\right) \\
& =\sum_{k=0}^{+\infty} T^{k} f(x) \int_{(k-\tau) / n}^{(k-\tau+1) / n} \varrho(s) d s .
\end{aligned}
$$

Таким способом из полученных выше результатов о неравномерном усреднении для полупотоков можно вывести новые способы усреднения для дискретного времени.

Автор благодарен профессору В. И. Богачеву за полезные обсуждения.

\section{СПИСОК ЦИТИРОВАННОЙ ЛИТЕРАТУРЫ}

[1] V. V. Kozlov, D. V. Treschev, J. Dynam. Control Systems, 9:3 (2003), 449-453. [2] В. В. Козлов, Д. В. Трещев, ТMФ, 136:3 (2003), 496-506. [3] В. И. Богачев, А. В. Королев, Докл. РАН, 412:3 (2007), 295-301. [4] V.I. Bogachev, Measure Theory, V. I, II, Springer-Verlag, Berlin, 2007. [5] В. И. Богачев, А. В. Королев, А. Ю. Пилипенко, Докл. РАН, 432:4 (2010), 439-442. [6] А. В. Королев, Укр. матем. журн., 62:5 (2010), 702-707. [7] И. П. Корнфельд, Я. Г. Синай, С. В. Фомин, Эргодическая теория, Наука, М., 1980. [8] U. Krengel, Ann. Inst. H. Poincaré Sect. B (N.S.), 12:4 (1976), 319-338. [9] N. Wiener, A. Wintner, Amer. J. Math., 63:4 (1941), 794-824. [10] N. Wiener, A. Wintner, Amer. J. Math., 63:2 (1941), 415-426.

\section{А. В. Королев}

E-mail: akorolevpro@mail.ru 